\title{
Article \\ Entropy Production as the origin of information encoding in RNA and DNA
}

\author{
Julián Mejía ${ }^{1, \dagger}$ and Karo Michaelian ${ }^{2 *}$ \\ 1 Postgrado in Physical Sciences, Instituto de Física, Universidad Nacional Autónoma de México, Cto. de la \\ Investigación Científica, Cuidad Universitaria, C.P. 04510, Mexico City, Mexico; julianmejia@ciencias.unam.mx \\ 2 Department of Nuclear Physics and Application of Radiation, Instituto de Física, Universidad Nacional \\ Autónoma de México, Cto. de la Investigación Científica, Cuidad Universitaria, C.P. 04510, Mexico City, Mexico; \\ karo@fisica.unam.mx \\ * Correspondence: karo@fisica.unam.mx \\ † Current address: Institut de Physique de Nice, 1361 Route des Lucioles, 06560 Valbonne, France
}

\begin{abstract}
Ultraviolet light incident on organic material can initiate its spontaneous dissipative structuring into chromophores which can catalyze their own replication. This may have been the case for one of the most ancient of all chromophores dissipating the Archean UVC photon flux, the nucleic acids. Oligos of nucleic acids with affinity to particular amino acids which foment UVC photon dissipation would have been selected through non-equilibrium thermodynamic imperatives which favor entropy production. Indeed, we show here that those amino acids with characteristics most relevant to fomenting UVC photon dissipation are precisely those with greatest chemical affinity to their codons or anticodons. Entropy production could thus provide an explanation for the accumulation of information in nucleic acids relevant to the dissipation of the externally imposed thermodynamic potentials. The accumulation of information in this manner provides a link between evolution and entropy production.
\end{abstract}

Keywords: entropy, entropy production, non-equilibrium thermodynamics, information encoding, nucleic acids, DNA, RNA, origin of life, origin of codons, amino acids, stereochemical era, photon potential

\section{Introduction}

Explaining the origin of the genetic code is a difficult problem since its resolution ultimately related with the origin of life itself. From the perspective of life as a fortuitous enzyme-catalyzed auto-catalytic reproductive event, the problem arises in that sufficient information must have somehow been acquired in the incipient genome before a necessarily faithful replication event could have taken place. Due to the complexity of such an event, involving denaturing, chiral selection, and extension, it is highly unlikely that that the sufficient information could have been generated at random.

A number of theories have attempted to addressed this information problem by considering the origin of the association between amino acids and their cognate codons or anticodons. The "stereochemical theory" suggests that amino acids became linked with their codons or anticodons based on stereo-chemical affinity[1-3], while the "frozen accident theory" [4,5] suggests that such links arose purely by chance but once established would have remained so since any changes would have been highly deleterious to protein construction and surely detrimental to the organism. The "co-evolutionary theory" [6] suggests instead 
that the structure of the codon system is primarily an imprint of the prebiotic pathways of amino-acid construction, which remain recognizable in contemporary enzymatic pathways of amino acid biosynthesis.

Such theories only partially address the problem of the origin of information in the genetic code since, since although they provide plausible reasons for the association between particular amino acids and their cognate codons, they do not suggest a physical-chemical basis for the specificity of such an association and how this relates to the origin of life itself; i.e. to enzyme-less replication, proliferation and evolution. Carl Woese [1] recognized this early on and emphasized this still unresolved problem of uncovering the basis of the specificity between amino acids and codons in the genetic code.

In this paper, by incorporating the stereochemical theory into the framework of non-equilibrium thermodynamic theory, we are able to provide a novel understanding of the physical-chemical basis of the specificity of these associations between codons/anticodons and amino acids. The basis we propose for this specificity is the suggestion that the the findamental molecules of life, and in particular the complexes of nucleic acid with amino acids, were formed through microscopic dissipative structuring under the Archean UVC solar photon spectrum prevailing at Earth's surface during the Archean to dissipate that spectrum into heat, i.e. to produce entropy [7-9]. We show that those complexes most efficient at photon dissipation would catalyze most efficiently their own reproduction, leading to a dissipation-replication relation. There is a non-equilibrium thermodynamic imperative which favors the amplification of fluctuations which lead to stationary states (disipative structures) with greater dissipation efficacy [10-16] . Information related to which nucleic acid - amino acid complexes provided most efficient photon dissipation would thus gradually have begun to be incorporated into the primitive genetic code.

\section{Foundations}

Clasical Irreversible Thermodynamic (CIT) theory, formulated by Lars Onsager $[10,11,17]$ and Ilya Prigogine [18] indicates that all irrevesible processes arise, persist, and even evolve to dissipate a generalized thermodynamic potential, i.e. to produce entropy. Glansdorff and Prigogine have shown how a non-linear system over which a generalized thermodynamic potential is imposed can "self-organize" into structures (or more correctly, processes) which can break symmetry in both space and time if this increases dissipation [13]. This organization of material is known as "dissipative structuring" in non-equilibrium thermodynamic language. Biologists are acutely aware of a sub-set of this structuring and refer to it simply as"life".

Macroscopic dissipative structuring leads to macroscopic processes such as hurricanes, winds, ocean currents and the water cylce. However, microscopic dissipative structuring can also occur [9], in which the structuring involves the molecular internal degrees of freedom; isomeric or tautomeric reconfiguration, rotation about covalent bonds, electric polarization or spin orientation in an external electric or magnetic field, electronic excited states, exciplex and excimer formation, etc.. In the case of structuring involving molecular configurational degrees of freedom, the structuring can remain even after the removal of the impressed generalized thermodynamic potential due to strong inter-atomic forces [9].

The most important generalized thermodynamic potential (source of free energy) at Earth's surface today, and also at the origin of life at $\sim 3.85 \mathrm{Ga}$, is the solar photon potential; the sun's spectrum containes a large amount of free energy with respect to the low free energy spectrum of the cosmic black-body radiation filling space surrounding Earth. During the Archean, sunlight surpassed all other forms of free energy (hydrothermal vents, lightening, chemical potentials, shock waves, etc.) by at least three orders of magnitude [19]. The most important dissipative structures existing on Earth's surface today are the organic pigments in water solvent [20] and these are responsible for approximately $63 \%$ of the total entropy production resulting from Earth's interaction with its solar environment [21]. The coupling of the heat of dissipation of the solar photons in organic pigments to other macroscopic irreversible processes 
such as ocean and wind currents and the water cycle, accounts for the majority of the rest of the entropy production on Earth [22].

Without the preexistence of complex biosynthetic pathways, the photon wavelengths that could have bootstrapped life through dissipation at life's origin in the Archean must have involved the long wavelength UVC and UVB regions, where there is enough free energy to make and break carbon covalent bonds, but not enough to fragment and thereby destroy organic molecules. A long wavelength UVC component (peaking at $\sim 260 \mathrm{~nm}$ ) of the solar spectrum indeed penetrated to the surface of Earth throughout the Archean with an integrated intensity of up to $5 \mathrm{~W} / \mathrm{m}^{2}$ midday at the equator $[7,23]$. Evolving life was exposed to this UVC photon potential for at least 1,000 million years $(1 \mathrm{Ga})$ until approximately $2.7 \mathrm{Ga}$ when organisms performing oxygenic photosynthesis became abundant enough to overwhelm the natural abiotic oxygen sinks [24].

Corroborating evidence for the exposure of life to this UVC photon potential, and indeed life's thermodynamic preoccupation with its dissipation, can be found in the fundamental molecules of life (those common to all three domains of life). Most of these absorb and dissipate this light into heat with great efficiency (see figure 1)[20,25]. We have therefore suggested that the fundamental molecules originated in the Archean as microscopic dissipative structures to perform this thermodynamic function $[7-9,25]$.

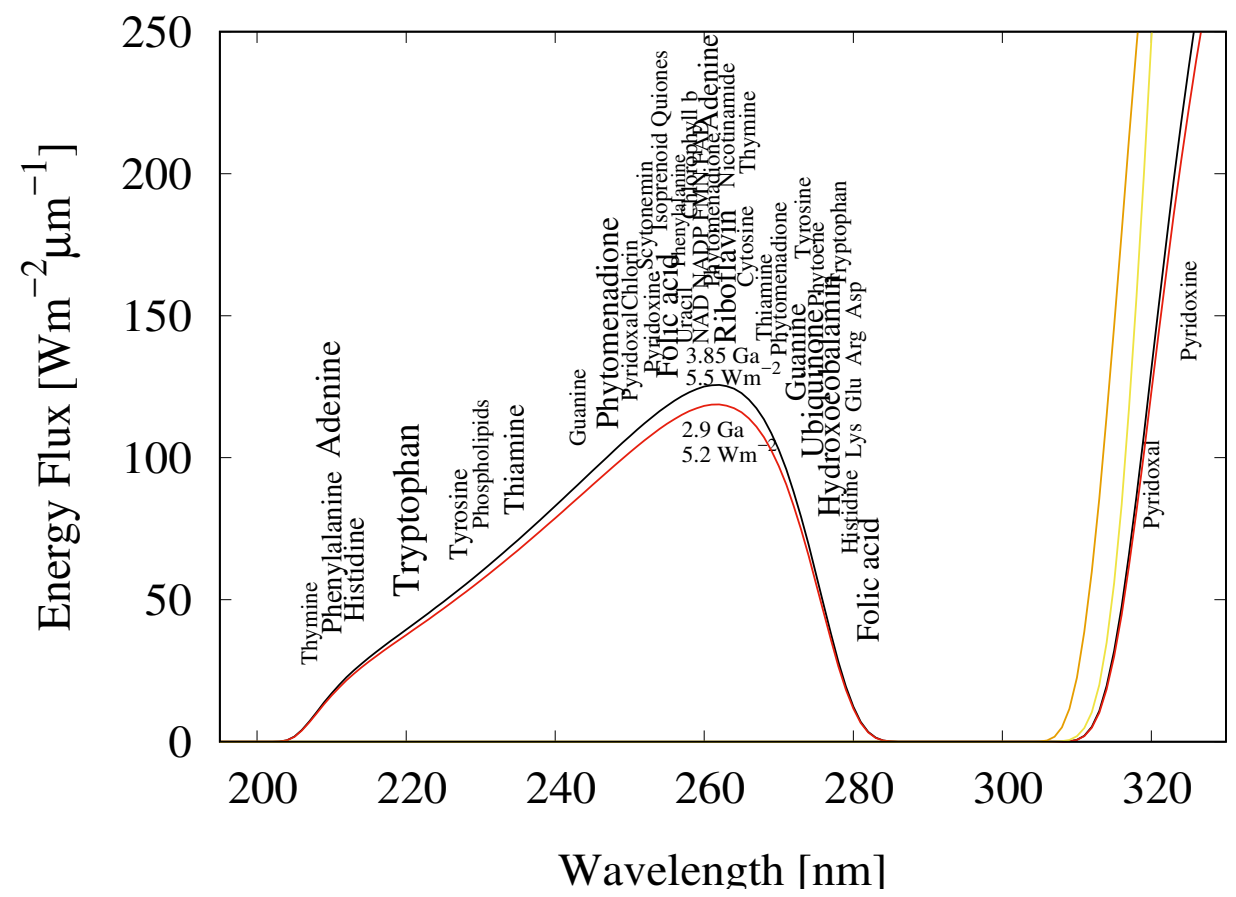

Figure 1. Fundamental molecules of life absorb in the UVC The wavelengths of maximum absorption of many of the fundamental molecules of life (common to all three domains), including the the nucleobases, aromatic and charged amino acids, cofactors, vitamins, and lipids coincide with the predicted solar spectrum at Earth's surface in the UVC at the time of the origin of life [23] around 3.85 Ga (black line) and until at least $2.9 \mathrm{Ga}$ (red line). The solar spectrum at Earth's surface today (green line) only has wavelengths greater than about $305 \mathrm{~nm}$ due to absorption by ozone in the upper atmosphere. The font size of the pigment name roughly indicates the relative size of its molar extinction coefficient. Adapted from [25]. 
The proliferation of these pigments over the whole surface of Earth, a hallmark of biological evolution, can be explained through the auto-catalytic nature of these pigments in dissipating the same UVC potential that produced them photochemically [25,26]. Analogously to what Prigogine [27] has demonstrated using CIT theory for auto-catalytic chemical reactions, the concentration of a photochemical product (chromophore or pigment) will increase much beyond its expected equilibrium concentration if the product acts as a catalyst for the dissipation the same impressed photon potential that produced it [26]. For fundamental chromophores of the Archean such as the nucleic acids absorbing in the UVC, this leads to a direct relation between dissipation and replication. Proliferation of these fundamental molecules in this manner over the whole of Earth's surface is thereby driven by the thermodynamic imperative of increasing the global entropy production of Earth in its solar environment. Although dissipative structuring of material may lead to a reduction in its entropy, that of the system plus environment invariably increases, thereby respecting the second law of thermodynamics.

Since microscopic dissipative structuring can be persistent, i.e. structuring remains even after the removal of the impressed external potential, information concerning the impressed potential and the molecular structuring required for its dissipation becomes programmed into the structure of the material. The cumulative historical information regarding the external generalized chemical potentials, and the blueprint for construction of the biosynthetic pathways needed for the production of structures (e.g. chromophores and their supporting structures) required for the dissipation of the externally imposed potentials, is today known as the genome. Such a microscopic mechanism with inherent persistence in structure, but also the production of variants through microscopic mutation of the genome, allows the system to "evolve", i.e. to dynamically adapt the dissipative structure towards optimal dissipation or to track changes in the external potential, or to resume dissipation should the external potential return after temporal absence (for example, after the overnight extinction of the solar photon potential).

The mechanism (to be described below) relating replication with dissipation, under the non-equilibrium thermodynamic imperative of increasing the global entropy production, allows the system to evolve to ever greater efficacy in photon dissipation (by, for example, increasing the areal coverage of pigments over Earth's surface, or increasing the wavelength region of dissipation) often with a corresponding increase in system complexity, eventually arriving at the complex global dissipative structure of today known as the biosphere which involves the coupling of biotic with abiotic dissipative processes [28]. Before describing this mechanisms in detail, we first discuss some of the salient properties of the fundamental molecules of life which provide evidence for, and are crucial to, the viability of such a mechanism.

\section{Salient properties of amino acid, nucleic acids, and their complexes}

\subsection{Absorption and dissipation}

The fundamental molecules of life (those molecules common to all three domains of life) and their associations in complexes are examples of microscopic self-organized dissipative structures which formed under the Archean UVC photon potential [7-9,25,26]. Evidence for this can be found in the strong absorption cross sections of the fundamental molecules, with absorption maxima lying exactly within the predicted UVC window of Earth's atmosphere during the Archean [23] (Fig. 1), and in the existence of inherent conical intersections doting these same molecules with extraordinarily rapid non-radiative excited state decay[20,29].

The nucleobases, and also their polymerization into single and double strand RNA and DNA, absorb and dissipate UVC light and can be formed from simpler precursor molecules such as hydrogen cyanide $\mathrm{HCN}$ in water by the very same UVC photons that they eventually dissipate with such efficacy $[9,20]$. 
An example of such microscopic dissipative structuring under UVC light is the generic photochemical pathway to the purine nucleobases first discovered by Ferris and Orgel in 1966 [30]. Although the particular photochemical reaction pathways to the multitude of other fundamental molecules (UVC pigments) still remain to be discovered, the existence of microscopic dissipative structuring is evidenced, for example, by the large quantity of organic pigment molecules found throughout the cosmos wherever UV light is available [31]. Today, a similar situation exists on Earth, with pigments dissipating in the near UV and visible which are constructed through more complex biosynthetic pathways but remain, nonetheless, microscopic dissipative structures dissipating the same visible light that provided the free energy for their production.

\subsection{Amino acid affinity to codons (or anti-codons) of DNA and RNA}

Stereochemical theories for the origin of the genetic code propose that chemical affinity between amino acids and nucleic acids was the historical basis for the present association between codons and/or anticodons and cognate amino acids [2,3,32-44]. These works present sufficient evidence to suggest that, at some early stage in the evolution of life, amino acids were directly associated with specific polynucleotides which later evolved into the genetic code.

Although evidence for the chemical association of the twenty common amino acids with RNA or DNA exists, not all associations are of the same character or strength. There are eight distinct types of associations between amino acids and nucleotides or their polymerizations into nuclei acids; 1) the $\pi$-cation bond[44,45], 2) $\pi$-stacking of aromatic structures [46], 3) Coulomb charge interaction [47], 4) hydrophobic aggregation [37], 5) Van der Waals interaction, 6) hydrogen bonding [48], 7) stereochemical (for example, substitution of nucleotides by amino acids of similar structure [39,49]), and 8) the possibility of amino acids synthesis from $\alpha$-ketos acids bound to dinucleotides [50].

Of these eight forms of association, four are non-specific, meaning that the amino acids do not bind to any particular sequence along the single or double strand DNA or RNA. However, the other four types of associations; the $\pi$-cation bonding, hydrogen bonding, stereochemical affinity and amino acid synthesis from $\alpha$-ketos, are specific, i.e. bind the amino acids almost unequivocally to either their codons or cognate anticodons.

One of the non-specific associations between nucleic acids and amino acids is due to the aliphatic nature of the side chain of the amino acids which can attach themselves, for example within the grooves of the nucleic acid, through van der Waals and hydrophobic interactions [3,43]. Another non-specific interaction is that between aromatic amino acids and nucleobases due to stacking interactions that occur when amino acid side chains contain charged sites like indole rings or aromatic rings, for example, Trp, Tyr, Phe and His [46]. Due to a favorable contribution from hydrophobic effects, stacking assumes an even greater importance in aqueous solution [48]. In addition, when the size of the indole or aromatic ring of the amino acid is similar to that of purine base, for example tryptophan, stacking may involve only one strand of the double helix RNA or DNA [34].

The types of specific interactions between aromatic amino acids and RNA or DNA are also varied. For example, as a result of the separation of the $\pi$-electrons from the nuclear charges in aromatic molecules, even if the electronic distribution is symmetric, a quadrapole electric moment arises. This allows the aromatic rings to interact as polar elements and form bonds such as $\pi$-cation. This distribution of charge in the aromatic ring confers high specificity to the bond produced between the aromatic amino acids and their cognate codons [44,45].

Possible recognition schemes for amino acids with charged residues are limited[48,49]. However, these amino acids can be bound to RNA or DNA because the $\alpha$-amino and $\alpha$-carboxyl groups provide 
good complements for hydrogen bond receptors and donors [44,48,49]. Amino acids which can also form a pair of hydrogen bonds with particular bases of nucleic acids are Asp, Glu, Asn and Gln [49].

An interesting example of specific stereochemical affinity between nucleotides and amino acids is related to their structural similarity. In fact, a nitrogenous base of DNA or RNA can be replaced by an amino acid of similar size while maintaining the structural integrity of the nucleic acid. The nucleobase which can be replaced correlates strongly with the second base of the cognate codon[39].

A hydrophobic relation has been established between all 20 common amino acids used by life and their codons or anti-codons. Codons having $\mathrm{U}$ as the second base have been associated with the most hydrophobic amino acids, and those having A as the second base are associated with the most hydrophylic amino acids [50]. Some of the found associations are highly specific and may have given rise to assignations for the homocodonic amino acids (Phe, Pro, Gly, Lys) [37,49,51].

An example of how photochemical reactions could also have played an important role in the later synthesis of amino acids can be found in the proposal that two bonded nitrogenous bases could have acted as catalysts for the synthesis of simple amino acid synthesis from $\alpha$-keto acids [50]. This could satisfactorily explain the hydrophobic properties of the simple amino acids and, at the same time, their affinity to their cognate codons (or at least the first two bases of the codon, a "di-codon"). The hydrophobic property would have allowed the complex to remain at the ocean surface where the UV flux was high and this hydrophobicity is inherited to the synthesized amino acid. Dinucleotides might thereby catalyze reactions required for the synthesis of amino acids by; providing free energy available through photon capture within the nucleotides [9], orientation and polarization of reactants through hydrogen bonding interactions, use of functional groups as nucleophiles or general bases, attachment of cofactors such as NADH or prebiotic equivalents, use of phosphate groups as an acid or a base catalyst, use of $\mathrm{Mg}^{+2}$ ions coordinated to phosphate groups as Lewis acid catalysts, and use of $\mathrm{Mg}^{+2}$-coordinated hydroxide ions as nucleophiles or general base catalysts [52-54]. UVC light interacting with nucleotides thus probably played a fundamental role in the early synthesis of these amino acids at some point during the Archean.

In summary, there exist an intrinsic and specific association between many of the amino acids and their cognate codons or anticodons. Except for the proposal of Copley et al. [50] for the synthesis of the amino acids employing catalytic dinucleotides, and the direct RNA template (DRT) theory for protein synthesis proposed by Yarus [2,44], the authors are not aware of any other theory explaining the specificity of association. The rest of this article describes our proposal for the chemical-physical basis of this specificity which is related to UVC photon dissipation and probably more relevant to the very beginnings of the origin of life, before peptides or the biosynthesis of amino acids were required.

\section{Ultraviolet and temperature assisted replication (UVTAR)}

This section describes the proposed mechanism by which replication of the RNA/DNA-amino acid complex can be associated with dissipation. First, however, we list some of the relevant ambient conditions of the Archean Earth surface.

The temperature of approximately $80-85^{\circ} \mathrm{C}$ of the ocean surface during the early Archean [55], when it is generally assumed that life arose, is strikingly similar to the short strand DNA melting temperatures, the temperature at which $50 \%$ of double strand DNA is denatured into single strands, in water at neutral $\mathrm{pH}$ and present ocean salinity. This provides an important clue as to the possible nature of enzyme-less reproduction at the origin of life.

The temperature at Earth's poles would have been colder, perhaps closer to the denaturing temperature of RNA under similar salt and $\mathrm{pH}$ ocean conditions $\left(40\right.$ to $\left.50^{\circ} \mathrm{C}\right)$, while the temperature at the equator would have been warmer. There would also have been a temperature profile with depth and a 
diurnal variation of temperature in the ocean microlayer similar to that of today, but probably of higher absolute temperature [20].

The Earth was cooling gradually as the greenhouse gas $\mathrm{CO}_{2}$ was being consumed in silicate carbonates formed through erosion of the newly forming continents [55], and once the local surface temperature fell below their respective denaturing temperatures, DNA and RNA single strand oligos (for example formed as dissipative structures through auto-catalytic photochemical and polymerization routes $[9,20]$ ) would eventually find and hydrogen bond with complementary oligos and normally be unable to separate again, thus preventing subsequent template reproduction. However, through absorption of UVC light during the day and dexcitation mechanisms such as proton transfer and the dissipation of this light into local heat, plus the absorption of solar infrared light on the ocean surface, "photon-induced denaturing" may have occurred. We have quantitatively measured this UVC-induced denaturing [56,57]. and it appears to be of sufficient size and to be reversible, i.e. most damage inflicted on the molecule by the UVC light is reversible and it can renature again without difficulty (see Fig. 2). Enzymeless denaturing is considered to be one of the most difficult problems for origin of life theories [58].

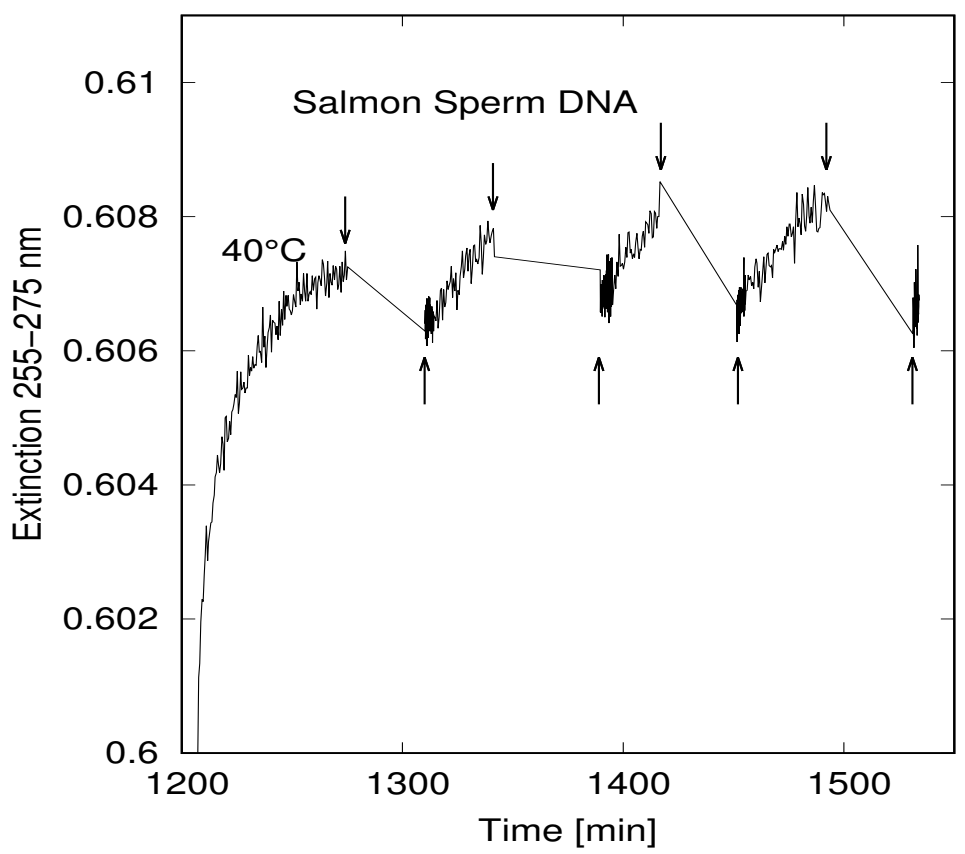

Figure 2. Experimental demonstration of UVC light-induced denaturing of DNA. Experimental demonstration of UVC light denaturing of Salmon sperm DNA of average length $100 \mathrm{Kbp}$ in pure water (without salt). The graph plots the extinction of the UVC light (due mainly to absorption with a small amount of scattering) by DNA in the wavelength range 255 to $265 \mathrm{~nm}$ against time as the UVC light was cycled on and off. The temperature of the bath was raised over a time period of 20 minutes to $40{ }^{\circ} \mathrm{C}$ and later maintained at this value $\left( \pm 0.01^{\circ} \mathrm{C}\right)$ for the duration of the experiment. The arrows pointing downward mark the time at which the UVC light was blocked from reaching the sample by a shutter and the arrows pointing upwards mark the times at which the light was allowed on sample by removing the shutter. It can be seen that while UVC light is on sample, the extinction increases gradually (after $1 / 2$ hour to about $0.3 \%$ of the differential absorption - between completely denatured and completely natured) due to the hypochromicity arising from UVC light-induced denaturing. While the light was blocked from the sample, the segments renatured, lowering the extinction. The amount of denaturing depends on the intensity of the UVC light and the temperature of the bath. Adapted with permision from Michaelian and an [57]. 
The characteristic steepness of the temperature denaturing curve for DNA, particularly for larger oligos and for acidic $\mathrm{pH}$ values $~ 5$ [59] would have facilitated photon-induced denaturing since, once the ambient temperature fell slightly below the melting temperature, because only the small amount of energy available in a single photon in the long wavelength UVC region $(\sim 4.8 \mathrm{eV})$ would have been sufficient to rupture many of the hydrogen bonds between the two strands and separate the strands completely. Double strand RNA has a lower melting temperature and a less steep denaturing curve. During overnight periods of approximately 7 hours (the Earth rotated more rapidly at the origin of life), the sharp denaturing curve of DNA would mean that the small decrease in ocean surface temperature would have been sufficient to allow for $\mathrm{Mg}^{2+}$ mediated extension of the separated single strands [58], completing the reproduction cycle (Fig 3).

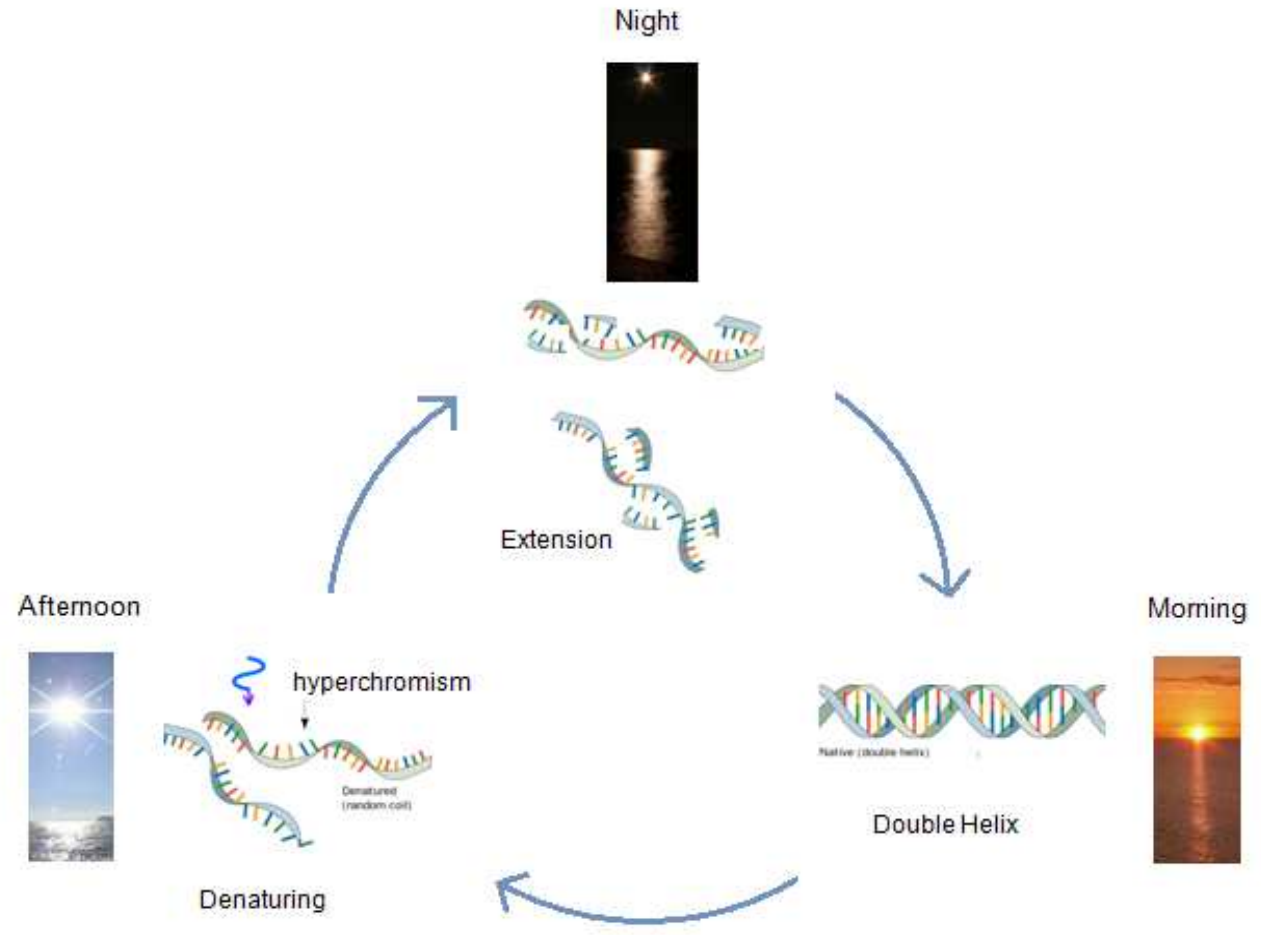

Figure 3. Ultraviolet and Temperature Assisted Reproduction (UVTAR) of RNA and DNA. UVTAR is a proposed mechanism based on photon dissipation for the enzyme-less reproduction of RNA and DNA assisted by the absorption of the prevailing UVC light flux and the high temperatures of the ocean surface during the Archean, including a day/night diurnal warming and cooling cycle of the ocean surface due to the absorption of solar infrared light. Most denaturing would occur in the afternoon when ocean surface temperatures were highest. "Hyperchromism" refers to the increase $(\sim 35 \%)$ in the absorption of photons at UVC wavelenghts $(\sim 260 \mathrm{~nm})$ once RNA or DNA are denatured into single strands. The important aspect of this mechanism is that replication is tied to dissipation, giving proliferation a thermodynamic imperative.

Single strand RNA and DNA is $20-40 \%$ (depending on length and sequence) more efficient at absorbing and dissipating UVC photons than is double strand RNA or DNA or the hybrid RNA+DNA duplex, an effect known as hyperchromism and is the result of the shadowing or screening of the bases when they are tightly stacked one above the other in a native double strand arrangement [60]. In single strand RNA and DNA, the bases are free to take on arbitrary orientation with respect to the long central 
axis of the RNA or DNA molecule and so there is less shadowing and therefore greater photon absorption. Denaturing by UVC light would thus increase the rate of dissipation of the solar photon potential in the UVC by about $20-40 \%$ compared to native double strand and, therefore, particular RNA and DNA that could have remained in the denatured state during the day through this mechanism of UVC absorption and dissipation and would have been available for template extension during the night, and thus, in this manner, be "thermodynamically" selected. If indeed this UVTAR mechanism were operative in the Archean, it would have endowed incipient life with a dissipation-replication relation, which, in fact, remains to this day in living systems and may be useful for placing evolutionary theory on a non-tautological physical-chemical foundation [20].

Enzymeless photon-induced denaturing is only one half of the complete enzymeless replication problem, the other half being extension (the formation of a new strand using the single strand as a template). However, there exists experimental evidence that extension of a complimentary strand can occur at high temperatures (around $80{ }^{\circ} \mathrm{C}$, similar to the surface temperature of the Archean) using chemically activated nucleotides in an aqueous solution containing $\mathrm{Mg}^{2+}$ or $\mathrm{Zn}^{2+}$ ions [58]. There also exists experimental data indicating that enzymeless DNA extension can be sped up orders of magnitude by including the presence of planer intercalating molecules, such as tryptophan, which prevent bending of the nucleic acid which hinders extension [61]. In our proposed UVTAR scenario, nucleotide activation would have been induced by the UVC light during daylight hours and extension would occur overnight when the ocean surface water had cooled by about $3-5^{\circ} \mathrm{C}$ and there would be no UVC light inhibiting the extension (Fig. 3). The ocean surface microlayer would have been rich in $\mathrm{Mg}^{2+}$ and $\mathrm{Zn}^{2+}$ ions and hydrophobic planer intercalating molecules [20].

Indications from our light-induced denaturing data [56,57], and this previously published data concerning enzymeless extension [58], are that a rudimentary enzymeless RNA or DNA replication driven by the thermodynamic imperative of photon dissipation would have been possible utilizing day-night UVC light cycling, under the physical conditions of Earth's ocean surface during the Archean (high UVC flux and high temperatures with $3-5^{\circ} \mathrm{C}$ diurnal cycling, and $\mathrm{pH}$ values of around 6-7).

\section{Accumulation of information through the dissipation-replication mechanism}

Being an irreversible thermodynamic process, life, since its origin, must have been coupled to the dissipation of a generalized chemical potential. We have explicitly identified this chemical potential at the origin of life in the Archean as the surface UVC photon potential. Furthermore, we have proposed an enzymeless ultraviolet and temperature assisted replication mechanism (UVTAR) powered by the dissipation of this light and leading to a dissipation-replication relation [7-9,20].

In the previous sections we have emphasized how the fundamental molecules of life are pigments which absorbed strongly the UVC light available at Earth's surface at the origin of life and throughout the Archean. We have also emphasized that the amino acids have affinity to RNA and DNA, and, in many cases, particularly to their specific codons or anticodons, evidence for a stereochemical era.

In the following subsections we discuss particular characteristics conferred to complexes of nucleic acids with amino acids which would promote the dissipation-replication relation and lead to greater photon dissipation. It is then shown that it is precisely these photon dissipation fomenting amino acids which have the strongest chemical affinity to their codons. We then show how this would have led to the accumulation of information within the nucleic acids relevant to each specific characteristic promoting photon dissipation of the complex. 


\subsection{Surface entrapment: amphipathic molecules}

Since water absorbs and reflects UVC light (1/e extinction depth of $\sim 1 \mathrm{~m}$ ) it would be crucial for optimizing dissipation and coupling to the water cycle that photochemical processes be carried out on the surface of Archean oceans where the organic molecules would have been exposed to the greatest UVC photon flux. Under normal circumstances, RNA and DNA would sediment to the ocean floor [56,57], rendering them useless for dissipation.

Eickbush and Moudrianakis [62] found that molecules intercalating RNA and DNA with an alkyl side arm which can fit into the major or minor groove could keep the nucleic acids at the surface. Even molecules like the polyamine spermidine, which are not intercalating but which can bind to the major or minor groove of DNA or RNA, thereby displacing water and making the molecule more hydrophobic, gives rise to entrapment at the surface. In fact, even the presence of simple mono- or divalent- cations saturating the primary or secondary ionic binding sites of RNA and DNA led to a low level of surface entrapment. Divalent $\mathrm{Ca}^{2+}$ and $\mathrm{NH}_{4}^{+}, \mathrm{Ba}^{2+}$ and $\mathrm{Na}^{2+}$ ions were found to induce entrapment at minimum concentrations about 30 times lower than that for monovalent ions. As with spermidine, the minimum cation concentration able to support entrapment corresponds to the concentration required to shield the negative phosphate charges [62].

Corroborating the suggestion that keeping RNA or DNA at the surface was fundamental to its thermodynamic function of photon dissipation, is the evidence that in the evolutionary sequence of amino acid coding, the first to be codified were most probably the hydrophobic amino acids [37,50]. The hydrophobic or non-polar amino acids are alanine (Ala), glycine (Gly), valine (Val), leucine (Leu), isoleucine (Ile), proline (Pro), methionine (Met), phenylalanine (Phe), and tryptophan (Trp). According to Trifonov [63], the early codon table involved only a few aminoacids; Ala, Asp, Gly, Pro, Ser, Thr and Val since these seven amino acids are encoded today by single point mutation derivatives of the presumed earliest parental codon GCU for alanine (Ala), which is a hydrophobic amino acid, as are Gly, Pro and Val[63] (Table 1). There is also a long-recognized relationship between the hydrophobicity of the amino acid and the second base of its codon. Copley et. al [50] suggest that this relationship can be explained if, before the emergence of complex biosynthetic pathways, simple amino acids were synthesized from $\alpha-$ keto acid precursors covalently attached to dinucleotides that catalyze the reactions required to synthesize the specific amino acid. 
Table 1. Attributes of the common amino acids classified by hydrophobicity and by electrostatic charge.

\begin{tabular}{|c|c|c|c|c|c|c|c|c|}
\hline Amino acid & Abbreviation & Codon & $\begin{array}{c}\text { Codon/Anticodon } \\
\text { Affinity }\end{array}$ & Amphipathic & $\begin{array}{l}\text { Antenna } \\
260 \mathrm{~nm}\end{array}$ & Intercalating & Catalysis & $\begin{array}{c}\text { Charge } \\
\text { Neutralizing }\end{array}$ \\
\hline \multicolumn{9}{|c|}{ Aliphatic not polar R group (Hydrophobics) } \\
\hline Glycine & Gly & $\begin{array}{l}\text { GGU } \\
\text { GGC } \\
\text { GGA } \\
\text { GGG }\end{array}$ & & & & & & \\
\hline Alanine & Ala & $\begin{array}{l}\text { GCU } \\
\text { GCC } \\
\text { GCA } \\
\text { GCG }\end{array}$ & & & & & & \\
\hline Proline & Pro & $\begin{array}{l}\text { CCU } \\
\text { CCC } \\
\text { CCA } \\
\text { CCG }\end{array}$ & & & & & & \\
\hline Valine & Val & $\begin{array}{l}\text { GUU } \\
\text { GUC } \\
\text { GUA } \\
\text { GUG }\end{array}$ & & & & & & \\
\hline Leucine & Leu & $\begin{array}{l}\text { UUA } \\
\text { UUG } \\
\text { CUU } \\
\text { CUC } \\
\text { CUA } \\
\text { CUG }\end{array}$ & & & & & & \\
\hline Isoleucine & Ile & $\begin{array}{l}\text { AUU } \\
\text { AUC } \\
\text { AUA }\end{array}$ & $\begin{array}{l}\mathrm{s} / \\
\mathrm{s}\end{array}$ & & yes & & & \\
\hline Metionine & Met & AUG & & yes & yes & & & \\
\hline \multicolumn{9}{|c|}{ Aromatic R group (Slightly hydrophobic) } \\
\hline Phenylalanine & Phe & $\begin{array}{l}\text { UUU } \\
\text { UUC }\end{array}$ & $/ \mathrm{m}$ & & yes & yes & & \\
\hline Tyrosine & Tyr & $\begin{array}{l}\text { UAU } \\
\text { UAC }\end{array}$ & $\begin{array}{l}/ \mathrm{s} \\
/ \mathrm{w}\end{array}$ & yes & yes & yes & & \\
\hline Tryptophan & $\operatorname{Trp}$ & UGG & $/ \mathrm{s}$ & yes & yes & yes & & \\
\hline \multicolumn{9}{|c|}{ Polar R group without charge } \\
\hline Serine & Ser & $\begin{array}{l}\text { UCU } \\
\text { UCC } \\
\text { UCA } \\
\text { UCG } \\
\text { AGU } \\
\text { AGC }\end{array}$ & & & & & & \\
\hline Threonine & Thr & $\begin{array}{l}\text { ACU } \\
\text { ACC } \\
\text { ACA } \\
\text { ACG }\end{array}$ & & & & & & \\
\hline Cysteine & Cys & $\begin{array}{l}\text { UGU } \\
\text { UGC }\end{array}$ & & & & & & \\
\hline Aspargine & Asn & $\begin{array}{l}\text { AAU } \\
\text { AAC }\end{array}$ & & & & & & \\
\hline Glutamine & Gln & $\begin{array}{l}\text { CAA } \\
\text { CAG }\end{array}$ & & & & & & \\
\hline \multicolumn{9}{|c|}{ R group positively charged } \\
\hline Lysine & Lys & $\begin{array}{l}\text { AAA } \\
\text { AAG }\end{array}$ & $/ \mathrm{s}$ & yes & yes* & & yes & yes \\
\hline Histidine & His & $\begin{array}{l}\text { CAU } \\
\text { CAC }\end{array}$ & $\begin{array}{l}/ \mathrm{w} \\
/ \mathrm{s}\end{array}$ & & yes* & yes & yes & yes \\
\hline Arginine & Arg & $\begin{array}{l}\text { CGU } \\
\text { CGC } \\
\text { CGA } \\
\text { CGG } \\
\text { AGA } \\
\text { AGG }\end{array}$ & $\begin{array}{l}/ \mathrm{s} \\
\mathrm{w} / \\
\mathrm{s} /\end{array}$ & & yes* & & yes & yes \\
\hline \multicolumn{9}{|c|}{ R group negatively charged } \\
\hline Aspartic acid & Asp & $\begin{array}{l}\text { GAU } \\
\text { GAC }\end{array}$ & & & yes* & & yes & \\
\hline Glutamic acid & Glu & $\begin{array}{l}\text { GAA } \\
\text { GAG }\end{array}$ & & & yes* & & yes & \\
\hline
\end{tabular}

Those amino acids having a strong (s; probability for non-specificity, Corr $P<10^{-4}$ ) or moderate ( $\mathrm{m}$; Corr $P<10^{-3}$ ) or weak ( $w$; Corr $P<10^{-2}$ ) association of their binding sites with their cognate codons/anticodons (Corr $P=1$ implies no specificity; see table 1 of [44]) are also those that have the greatest number of characteristics relevant to increased photon dissipation, and therefore reproduction through the dissipation-replication relation, at the origin of life. The "*" indicates amino acids that absorb in the $260 \mathrm{~nm}$ window through charge transfer (CT) transitions [64]. 
We suggest here that at the very beginnings of life, amphiphatic molecules, having both hydrophylic and hydrophobic parts, that can adsorb to DNA or RNA and thereby keep them afloat at the surface would have been essential to photon dissipation. The amphiphatic amino acids are; lysine, methionine, tryptophan, tyrosine (Table 1). The amphiphatic amino acids were thus very important to the prevention of sedimentation to the seabed thereby securing the efficacy in photon dissipation of the RNA/DNA amino acid complex.

\subsection{Charge neutralization}

It has been suggested that salt concentrations in the Archean would have been 1.5 to 2 times greater than present day ocean concentrations due to the likelihood that salt entrapment basins at sea shores would have been limited due to late formation of continents [65] and the fact that bacterial mats preventing salt erosion back into the ocean at these sites [66] would not have existed. At the high ocean surface temperatures of the Archean, particularly near to the equator, neutralization of the negative charges on the RNA or DNA backbone by either salt ions or positively charged amino acids would have allowed overnight extension to occur at higher surface temperatures than otherwise. The positively charged amino acids would have permitted a UVTAR mechanism to be viable in environments of lower salt concentration. The amino acids histidine, lysine and arginine have net positive charge and may thus have been important to replication at higher temperatures in these low salt environments such as estuaries.

Charge neutralization of the negatively charged phosphates on RNA or DNA could be achieved by molecules which bind ionically and fit within the major or minor grooves, such as spermadine mentioned in the previous subsection or the positively charged amino acids with hydrophilic side chains. However, over saturation of the sites can lead to precipitation of RNA or DNA so there exists a narrow concentration range of such molecules supporting surface entrapment [62]. Molecules which were both charge neutralizing and hydrophobic would have performed best at keeping RNA or DNA at the surface and able to template replicate at the high ocean surface temperatures of the Archean. Of the amphiphatic amino acids (see previous subsection), only lysine is positively charged. In this regard, it is interesting the lysine is highly specifically bonded to its anticodon and is a homocodonic amino acid $[37,49,51]$.

\subsection{Antenna molecules}

The nucleobases of RNA and DNA have large molar extinction coefficients for light in the UVC wavelength region and are extremely rapid in the dissipation of their electronically excited singlet states to the ground state, which happens on sub-picosecond time scales through a conical intersection. Also very rapid ( $\sim 2 \mathrm{ps}$ ) is the vibrational cooling of the hot molecule to the temperature of its water solvent environment [29].

The aromatic amino acids also have strong absorbance in the UVC region, however, their excited state lifetimes are on the order of nanoseconds, or about three orders of magnitude longer than the excited state lifetimes of the nucleic acids and they have a significant quantum efficiency for radiative decay through flourescence $[67,68]$.

The natural stacking affinity existing between aromatic amino acids and nucleic acids keeps them close enough to permit excitation energy transfer (EET), allowing the energy of electronic excitation of the amino acid to be dissipated non-radiatively through the conical intersection of the RNA or DNA. Evidence of energy transfer between tryptophan and DNA exists in the observation that the fluorescence of tryptophan is completely quenched when nucleobases or nucleosides are included in solution [69-72]. The amino acid acts as a donor antenna molecule to the acceptor quencher RNA or DNA molecule, increasing the photon dissipation efficacy of the complex over what the components acting separately could achieve. 
The aromatic amino acids are histidine, phenylalanine, tryptophan, and tyrosine. Based on the strength of binding to their codons/anticodons, Yarus et al. [44] identify these amino acids as among the best candidates for participation in a stereochemical era based on amino acid binding at cognate codon or anticodon sites (Table 1). Contrary to the notion that these aromatic amino acids must have appeared much later in the history of life because of the complexity of their present biosynthetic pathways, UVC light offers a unique energy source for producing aromatic carbon based structures and we present evidence for this in the Discussion section.

The binding of aromatic amino acids as antenna molecules to oligonucleotides would foment the dissipation-replication relation by increasing UVC-induced denaturing. Thus, oligos with affinity to aromatic amino acids would have a higher probability of UVTAR replication than random nucleotide sequences with no such affinity. In this manner the affinity between codons and amino acids is preserved in the sequence of the codon by what could be called "thermodynamic selection" on dissipation.

\subsection{Intercalation}

Intercalating molecules have been shown to increase the rigidity of RNA and DNA strands, thereby inhibiting cyclization which hinders enzymeless extension [73]. In fact, the efficiency for enzymeless extension in the presence of intercalating molecules can be improved by orders of magnitude [61]. To fit between the bases, these intercalating molecules must be planer aromatic and have important overlap with the bases for strongest non-covalent binding.

Tryptophan has a large UVC absorption cross section but a slow decay time of nanoseconds and high yield for fluorescence [74]. The size of its ring structure is similar to that of the bases so it intercalates strongly and can remain attached even to single strand nucleic acid [34]. Therefore, during the daytime in the Archean it could have acted as an antenna molecule passing its excited state energy to RNA and DNA through resonant energy transfer, inhibiting fluorescence. At night, tryptophan could have acted as an extension enhancer.

Peptides containing a planer moiety are also candidates for intercalation, for example the tripeptide LysTrpLys which intercalates through a two step mechanism; first electrostatic binding between the positively charged Lys residues and the negatively charged phosphate group of the backbone and then stacking of the indole moeity of the Trp residue [72]. Such a two step binding process has also been observed for other combinations of charged and aromatic amino acids such as LysPheArg [75]. Both of these tripeptides, with confirmed affinity to their string of cognate anticodons, retain physiological function in contemporary enzymes.

\subsection{Catalysis}

Another amino acid with a strong affinity to its anticodon is histidine [44]. This is an 5-member aromatic heterocycle containing two nitrogen atoms and 3 carbon atoms known as an imidazole. Having two conjugate bonds, histidine absorbs strongly in the UVC $(212 \mathrm{~nm}, \epsilon=5,700)$ [76], however, it has a smaller absorption peak at $280 \mathrm{~nm}$ which has been attributed to photon-induced charge transfer (CT) transitions [64]. This could be important to the origin of life because imidazoles are known as amphoteric molecules, serving as both an acidic and alkaline $(\mathrm{pKa}=14.5)$ catalyst for a very large number of important reactions in contemporary life, for example as a condensing agents for the phosphorylation of the nucleobases and the lipids.

The stacking of the aromatic rings of histidine with, for example, the imidazole rings of the purine bases, could lead to yet another type of catalysis by, for example, the heat generated from the photon dissipation in histidine catalyzing the subsequent thermal chemical reactions required in purine base production, for example, in the case of the microscopic dissipative structuring of Adenine from HCN [9]. 
Other non-aromatic amino acids that have strong absorption in the UVC Archean atmospheric window region $(\sim 280 \mathrm{~nm}$ ) attributed to charge transfer transitions, are the charged amino acids Lys, Glu monosodium salt (Glu·Na), Arg, and Asp potassium salt (Asp·K) [64]. According to Yarus et al. [44] these, along with histidine, have strong association with either their cognate codons or anticodons (Table 1). These, therefore, by dissipating the photon energy through charge transfer, could have acted as catalysts for early biotic photochemistry such as phosphorylation of the nucleotides required for extension.

\section{Amino acids which promote the dissipation-replication relation and their affinity to codon/anticodon}

In Table 1 we compare the amino acids with the above listed characteristics which would have promoted entropy production and the dissipation-replication relation through the UVTAR mechanism with the the strength of association between an amino acid and their cognate condons or anticodons as determined by Yarus and coworkers [44]. It is striking that those amino acids with those characteristics mentioned above that could most enhance the dissipation-replication relation, through one or more of the above mentioned properties, are precisely those with strongest association with their anticodons or codons.

We suggest that these characteristics are therefore the basis of the specificity of the interaction between nucleic acids and amino acids. Optimizing the dissipation of the long wavelength UVC region of the Archean solar spectrum could thus have been the origin of the information encoding in RNA and DNA. This is still relevant for the genomes of today's organisms which are essentially blueprints for the construction of complex biosynthetic pathways to dissipate the external generalized thermodynamic potentials existent (or once existent) in the organisms environment.

\section{Discussion}

The properties of amino acids which may have been important to enhancing dissipation are; their amphiphatic character required for entrapment of RNA and DNA at the ocean surface, their UVC light antenna properties for greater photon collection to foment denaturation, intercalating facility to enhance energy transfer for rapid dissipation and overnight extension as well as reduce photoproducts, charge neutralizing properties to foment replication at earlier higher temperatures in low salt environments, and catalytic properties (e.g. charge transfer) to foment chemical reactions required for replication.

Yarus and coworkers [44] building on the work of others [37,49,51] have unequivocally demonstrated that there exists a chemical affinity for a subset of amino acids to sites containing their cognate codons or anticodons, and that this is indicative of a stereochemical era near the beginning of life. Here we have shown that it is exactly these amino acids having affinity to their codons or anticodons that have the properties necessary for fomenting an efficient dissipation and enzymeless replication. Although the data of Yarus and coworkers was obtained with RNA, the codon scaffolding and affinity to amino acids is very similar for DNA.

It is sometimes argued that the aromatic amino acids must have appeared later in the evolutionary history of life due to their contemporary complex biosynthetic pathways. However, this ignores the relatively simple UV photochemical pathways to such molecules which could have existed throughout the whole of the Archean. For example, there are relatively few steps involved in the production of the even more complex nucleobase adenine starting from $\mathrm{HCN}$ in water [9]. If adenine was produced abiotically early in the history of life, then it is probable that the aromatic amino acids could also have been. Indeed, Cataldo [77] has suggested that the rings of the aromatic amino acids could be derived from UVC light acting on acetylene derived from methane and ethylene, ultimately derived from $\mathrm{CO}_{2}$ saturated water under UVC wavelengths. Aromatic hydrocarbons have also been found in meteorites [78] 
452

453

454

455

456

457

458

459

460

461

462

463

464

465

466

467

468

and interstellar space [31,79]. The stereochemical affinity of these to RNA and DNA [44] and the large number of characteristics these have which are useful for the proposed enzymeless replication of RNA and DNA (Table 1) argue instead for these being among the first amino acids important to incipient life.

It is also been argued by Copley et al. [50] that the present triplet code originated from a binary one in which the first two bases of the codon specified uniquely 14 of the 22 bases used by life and that this was related to the possibility of dinucleotides acting as catalysts for the production of the most simple amino acids from $\alpha$-keto acids. Although this hypothesis is appealing, and indeed we have suggested that intermediates on the way to the purines, such as imidazoles could have acted as photochemical catalysts for other fundamental molecules [9], there is no evidence for a stereochemical affinity between the dinucleotides and these suggested amino acids or their precursor $\alpha$-keto acids, except, perhaps for the negatively charged amino acids Glu and Asp. Furthermore, the contemporary chemical route to $\alpha$-keto acids is quite complex and these do not appear to have a photochemical origin. We therefore suggest that the associations suggested by Copley et al. may indeed have been some of the first steps on route to a purely chemical (independent of UVC photons) biosynthetic pathway of the simple amino acids, but that this probably appeared much later in the evolutionary history of life, i.e. much after the appearance of stereochemical associations discovered by Yarus et al. [44] and before the emergence and subsequent molecular evolution of translation ribosomes and enzymes [80].

\section{Conclusions}

We have suggested here that the first information programmed into the microscopic dissipative structures known as RNA and DNA had a direct thermodynamic utility; that of providing a physical scaffolding for the attachment of molecules which aid in the dissipation of the prevailing UVC solar photon potential. We postulate that this was achieved through a dissipation-replication relation for RNA and DNA employing an ultraviolet and temperature assisted mechanism for enzymeless replication (UVTAR) as presented in section 4. Since this mechanism associates replication with UVC photon dissipation, it provides a non-equilibrium thermodynamic and physical-chemical basis for the accumulation of information relevant to the origin and evolution of life as a dissipative process.

The other amino acids without properties relevant to the UVTAR process must have been relevant to reproduction at some point in the history of life and were probably assigned codons once metabolic pathways had been established in a more advanced coevolutionary process [6,33]. During coevolution, not only could amino acids have been added to the code, but earlier codonic assignments may have been changed. Despite this, it is important to emphasize that there are sequences of amino acids and their codonic assignments that have remained immutable since ancestral times [81,82].

Finally, we end with a quote by Carl Woese (1967) reproduced in the seminal paper of Yarus et al. [44] referenced frequently above;

"I am particularly struck by the difficulty of getting [the genetic code] started unless there is some basis in the specificity of interaction between nucleic acids and amino acids or polypeptide to build upon."

The work of Yarus and coworkers and others has demonstrated the specificity in the interaction between nucleic acids and amino acids beyond any reasonable doubt. Our work presented here is a proposal for the basis of that specificity; that of increasing photon dissipation efficacy (entropy production) of the RNA- and DNA-amino acid complexes which leads to higher differential replication through a UVTAR mechanism operating under non-equilibrium thermodynamic imperatives.

Author Contributions: conceptualization, methodology, validation, investigation, and writing the draft, K. Michaelian and J. Mejía; resources, supervision, project administration, funding acquisition, K. Michaelian. 
496 Funding: This research was funded by DGAPA-UNAM, project number IN102316, and the National 497 Quality Postgraduate Program CONACyT.

498 Conflicts of Interest: The authors declare no conflict of interest. The funders had no role in the design of the study; in 499 the collection, analyses, or interpretation of data; in the writing of the manuscript, or in the decision to publish the 500 results.

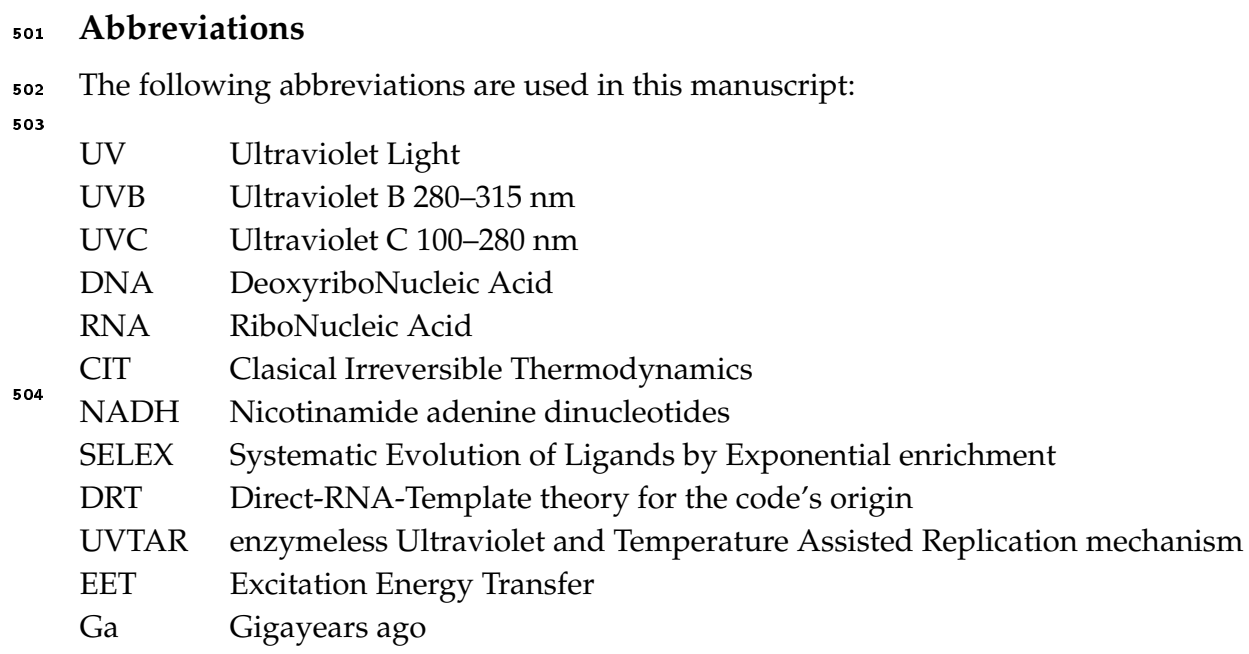

505 References

506 1. Woese, C.R. The genetic code: the molecular basis for genetic expression; Harper \& Row, 1967.

507 2. Yarus, M. Amino Acids as RNA Ligands: A Direct-RNA-Template Theory for the Codes Origin. J. Mol. Evol. $508 \quad$ 1998, pp. 109-17.

509 3. Yarus, M.; Caporaso, J.G.; Knight, R. Origins The Genetic Code: The Escaped Triplet Theory. Annu. Rev. $510 \quad$ Biochem. 2005, pp. 179-98.

511 4. Crick, F. The origin of the genetic code. Journal of Molecular Biology 1968, 38 , 367 - 379. 512 doi:https:// doi.org/10.1016/0022-2836(68)90392-6.

$5135 . \quad K o o n i n$, E.V. Frozen Accident Pushing 50: Stereochemistry, Expansion, and Chance in the Evolution of the $514 \quad$ Genetic Code. Life 2017, 7, 22.

515 6. Wong, J.T.F. Co-Evolution Theory of Genetic Code. Proceedings of the National Academy of Sciences of the United $516 \quad$ States of America 1975, 72, 1909-1912.

517 7. Michaelian, K. Thermodynamic origin of life. ArXiv 2009.

518 8. Michaelian, K. Thermodynamic dissipation theory for the origin of life. Earth Syst. Dynam. $2011,224,37-51$.

519 9. Michaelian, K. Microscopic Dissipative Structuring and Proliferation at the Origin of Life. Heliyon 2017, $520 \quad 3$, e00424. doi:10.1016/j.heliyon.2017.e00424.

521 10. Onsager, L. Reciprocal Relations in Irreversible Processes, I. Phys. Rev. 1931, 37, 405-426.

522 11. Onsager, L. Reciprocal Relations in Irreversible Processes, II. Phys. Rev. 1931, 38, 2265.

523 12. Prigogine, I.; Nicolis, G. Biological order, structure and instabilities. Quarterly Reviews of Biophysic 1971, $524 \quad 4,107-144$.

525 13. Glansdorff, P.; Prigogine, I. Thermodynamic Theory of Structure, Stability and Fluctuations; Wiley - Interscience., $526 \quad 1971$

527 14. Gaspard, P., Engineering of Chemical Complexity; World Scientific, 2013; chapter Self-Organization at the

15. Gaspard, P. Fluctuation theorem for nonequilibrium reactions. The Journal of Chemical Physics 2004, 530 120, 8898-8905, [https://doi.org/10.1063/1.1688758]. doi:10.1063/1.1688758. 


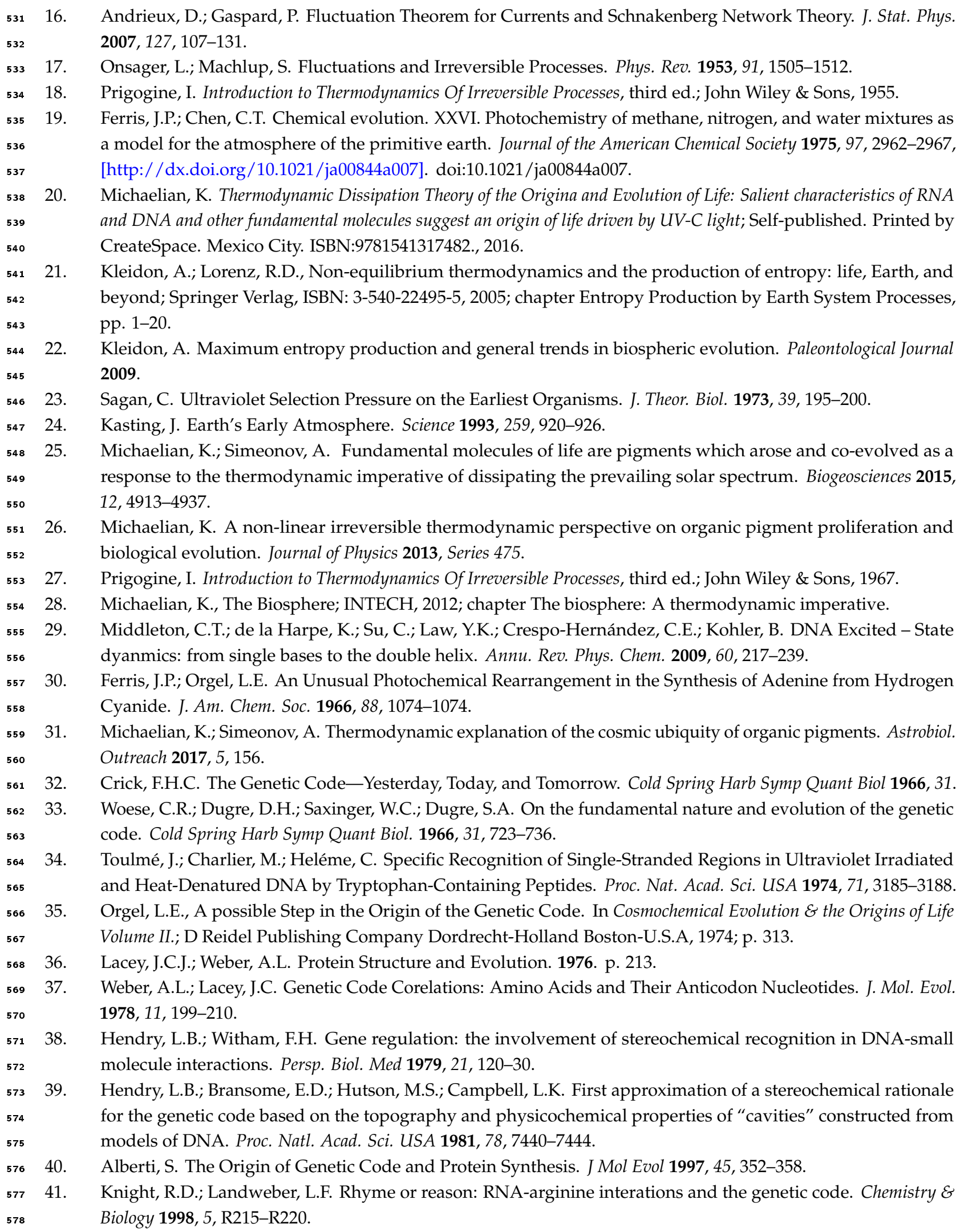




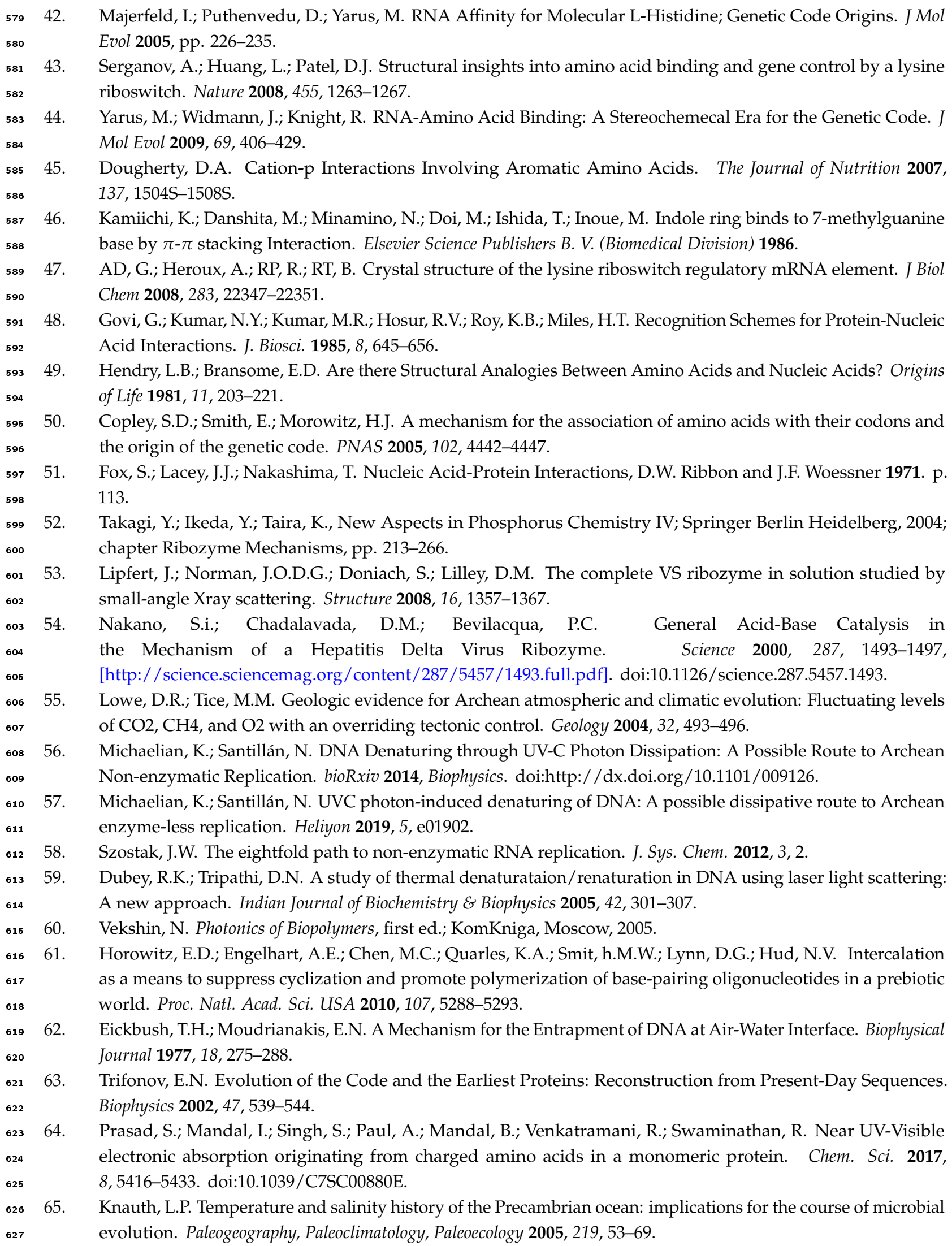




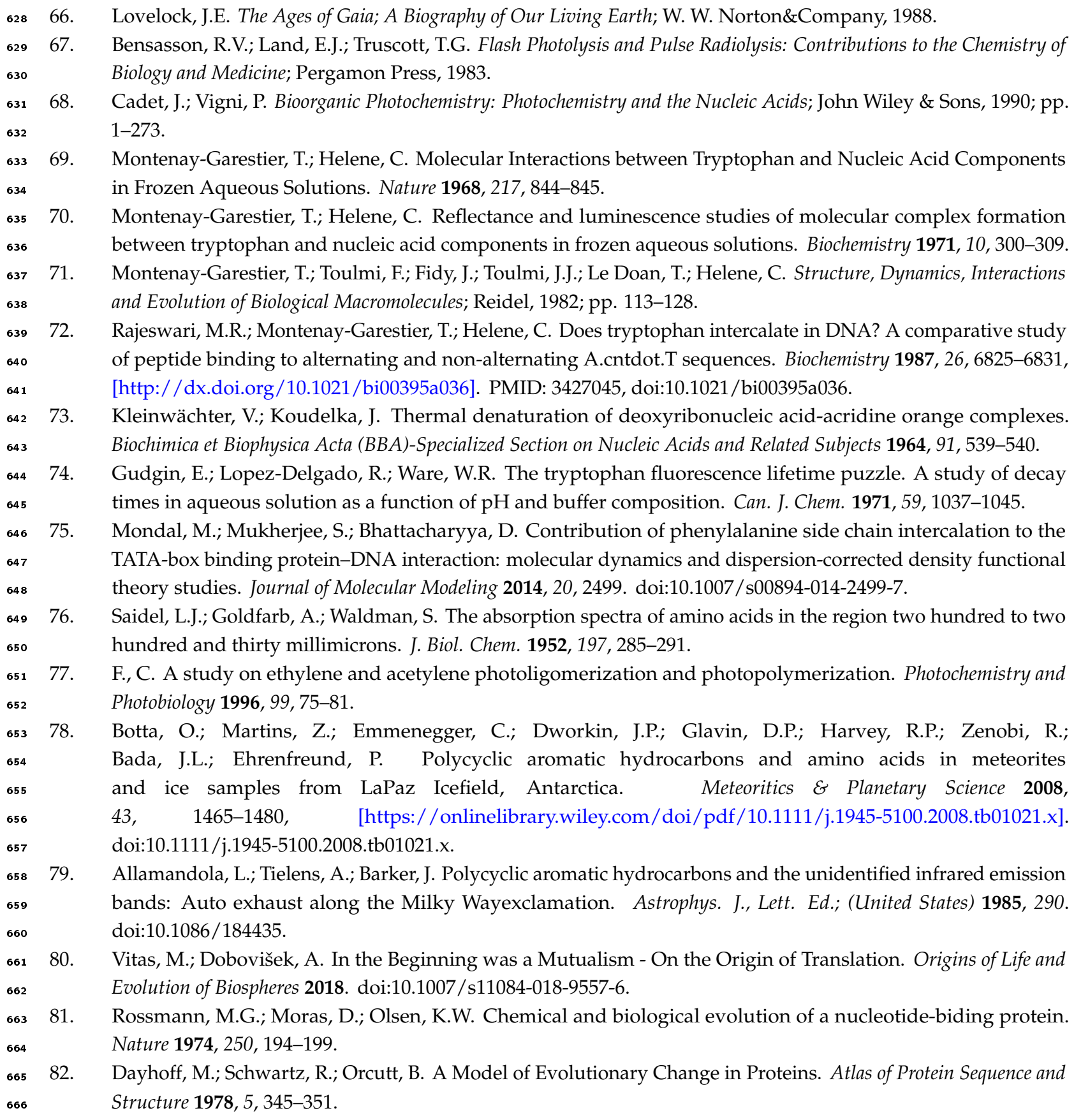

